\title{
QUANTIFICAÇÃO E QUALIFICAÇÃO DE PRODUTOS GERADOS NO PROCESSO ONDATEC DE PRODUÇÃO DE CARVÃO VEGETAL POR MICRO-ONDAS*
}

\section{Resumo}

Anderson dos Santos Morais ${ }^{1}$ Tiago LuisOliveira ${ }^{2}$

Paulo Santos Assis ${ }^{3}$ Alessandra Chagas Daniel ${ }^{4}$ Marco Aurélio Dumont Porto Thales Eduardo Leal ${ }^{6}$ Renan Cezar Artilha ${ }^{7}$ Lucas Gibram Leite e Silva

Em função da crise energética e do aquecimento global, faz-se necessário a utilização de novas fontes de energia como uso sustentável da matriz energética disponível. Do exposto, a CEMIG GT, a ONDATEC e a Universidade Federal de Ouro Preto, investiram em um projeto no âmbito do Programa (P\&D) Cemig/Aneel para geração de energia. O objetivo deste trabalho foi efetuar a avaliação do potencial energético dos efluentes gasosos (gases não condensáveis - GNC) e líquidos (Gases Condensáveis) de uma planta de pirólise de biomassa via micro-ondas para a produção de carvão vegetal. Para tanto, a ONDATEC construiu uma unidade piloto de produção de carvão vegetal (pirólise) por micro-ondas com capacidade de produção de $150 \mathrm{~kg}$ por hora. O processo de pirólise ocorreu em modo contínuo e em dois estágios, a secagem da biomassa e a pirólise propriamente dita, com a produção de carvão vegetal, gás de pirólise (GNC) e bio-óleo (GC). Em face dos resultados obtidos para o GC e o GNC, foi verificado um potencial de geração de 2,24 MWh de energia elétrica por tonelada de carvão produzido, que pode ser uma boa alternativa para desonerar a cadeia produtiva do carvão vegetal e do ferro gusa.

Palavras-chaves: Biomassa; Pirólise; Micro-ondas; Energia.

\section{MEASUREMENT AND QUALIFICATION OF GASES GENERATED IN THE PROCESS OF CHARCOAL PRODUCTION IN MICROWAVE TECHNOLOGY - BY ONDATEC COMPANY Abstract}

Facing energy crisis and global warming, the use of new energy sources such as sustainable ones has become imperative. In this context, CEMIG GT Company, ONDATEC Company and the Federal University of Ouro Preto, invested in a project under the Program (R\&D) CEMIG/ANEEL for power generation. The objective of this work was to test the energy potential of waste gases (non-condensable gases-NCG) and liquid (condensable gases-CG) of a biomass pyrolysis plant by microwave to produce charcoal. Therefore, ONDATEC built a pilot unit by microwave charcoal production (pyrolysis) microwave with a capacity of $150 \mathrm{~kg}$ per hour. The pyrolysis process was in a continuous mode and in two stages: the biomass drying and the pyrolysis itself. As a result, charcoal, pyrolysis gas (NCG) and bio-oil (CG) were produced. According to the results obtained for the CG and the NCG, it was found a generation potential of $2.24 \mathrm{MWh}$ of electric energy produced by one ton of charcoal, which can be a good alternative to relieve the production chain of charcoal and pig iron.

Keywords: Biomass; Pyrolysis; Microwave; Energy.

\footnotetext{
Engenheiro de Alimentos, Doutor em Engenharia Química, Ondatec - Tecnologia Industrial em micro-ondas, Uberaba, MG, Brasil.

Eng. Mecânico, Doutorando em Engenharia de Materiais, Redemat, UFOP, Ouro Preto, MG, Brasil.

Engenheiro Metalurgista, M.Sc., Dr.-Ing. Professor Titular da Escola de Minas, UFOP, Diretor da ABM, Professor da REDEMAT, Ouro Preto, MG, Brasil.

4 Engenheira Química, Química, Tec. Tecnologia e Normalização, CEMIG, gerente do projeto, Belo Horizonte, $M G$, Brasil.

Eng. Civil, Gerente de Alternativas Energéticas, CEMIG, Belo Horizonte, MG, Brasil.

Eng. Metalurgista, Mestrando em Engenharia de Materiais, Redemat, UFOP, Ouro Preto, MG, Brasil.

Graduando em Engenharia Metalúrgica, UFOP, Ouro Preto, MG, Brasil.

Eng. Metalurgista, Mestrando em Eng. de Materiais, Redemat, UFOP, Ouro Preto, MG, Brasil.
} 


\section{INTRODUÇÃO}

A evolução do consumo mundial de energia, baseada em combustíveis fósseis, conduziu a humanidade para uma matriz energética cara e, sobretudo, bastante negativa para o meio ambiente [1]. A demanda mundial por energia tem sido suprida por fontes não renováveis (82\%) e fontes renováveis $(18 \%)$ [2].

Thiago et alii (2010) afirma que em 2010, 47\% da energia do Brasil foi proveniente de fonte renovavel, sendo que $30 \%$ deste valor correspondeu a energia proveniente do uso de biomassa [3].

De acordo com o $28^{\circ}$ Balanço Energético do Estado de Minas Gerais [5] a demanda total da participação da lenha no estado foi de $20,3 \%$ em 2012. O setor industrial foi o maior consumidor do carvão distribuído. A indústria não integrada de ferro gusa consumiu $45,6 \%$ do carvão destinado ao setor. Isso se deve principalmente à resentatividade das siderurgias em Minas Gerais, que possui grandes consumidores de carvão vegetal.

Braga afirma que a pirólise da madeira produz carvão vegetal, tiços, gases condensáveis (licor pirolenhoso e alcatrão vegetal) e gases não condensáveis $\left(\mathrm{CO}_{2}, \mathrm{CO}, \mathrm{CH}_{4}, \mathrm{H}_{2}, \mathrm{O}_{2}, \mathrm{~N}_{2}\right.$ e hidrocarbonetos). De acordo com o autor os gases efluentes podem ser utilizados para melhorar a eficiência térmica do processo de obtenção de carvão vegetal, secagem da madeira, cogeração e diminuição das emissões de gases de efeito estufa [4].

Segundo Pereira, os fornos de micro-ondas tem participado com parcelas cada vez maiores do mercado, como alternativa para a pirólise da madeira. Contudo, o uso da energia de micro-ondas, ainda é visto com restrição pelos empresários, que relutam em adotar a tecnologia, seja por receio de uma rejeição dos seus produtos, ou pelo custo elevado de implantação do sistema [6].

Pereira afirma que o uso da energia de micro-ondas para aquecimento de materiais dielétricos, começou a ganhar impulso em 1946 pelos engenheiros da Raytheon Corporation. No Brasil apenas na década de 90, os fornos de microondas passaram a fazer parte do cotidiano dos consumidores [6].

Fernández afirma que a pirólise por micro-ondas é uma atrativa alternativa tecnológica sobre os processos convencionais de pirólise, sob o ponto de vista da velocidade de processamento, bem como da qualidade dos produtos obtidos [7].

De acordo com Fernández quando o aquecimento por micro-ondas é empregado na pirólise, ocorre uma melhora na distribuição da temperatura, na taxa de aquecimento e no tempo de residência dos voláteis em comparação com o processo convencional de pirólise [7].

Considerando a madeira como biomassa para produção de energia, torna-se necessário levar em consideração várias características, como sua composição química, elementar e imediata. Além destas, propriedades como densidade básica, teor de umidade, poder calorífico inferior, superior e líquido são de considerável relevância [8]. A lignina é um componente aromático rico em carbono e de alta estabilidade térmica com poder calorífico em torno de 6.400 $\mathrm{cal} / \mathrm{g}$. Os extrativos podem apresentar elevado poder calorífico, chegando a até $8.300 \mathrm{cal} / \mathrm{g}$. O teor de minerais afeta negativamente o valor calórico do combustível, sendo, portanto, indesejável [8]. Em relação a sua composição química, a madeira madura é formada por uma mistura de polímeros, 
constituída basicamente por carboidratos (holocelulose), lignina, extrativos e elementos minerais, em que os teores de lignina e de extrativos são de grande relevância energética $[9,10]$.

Nos processos termoquímicos, os hidrocarbonetos presentes na madeira, principalmente a celulose, a hemicelulose e a lignina, sofrem uma série de reações de decomposição térmica gerando GNC, GC e produzindo carvão. Os produtos desta decomposição variam em função da temperatura, pressão e composição do gás durante a desvolatização, sendo que o processo de pirólise começa quando componentes termicamente instáveis são quebrados e evaporam com outros voláteis.

Quanto aos GNC, esses são constituídos principalmente de $\mathrm{CO}, \mathrm{CH}_{4}$ e quantidades menores de hidrocarbonetos, além de $\mathrm{H}_{2}$ e $\mathrm{CO}_{2}$, sendo que 0 rendimento de $\mathrm{CO}$ e $\mathrm{CO}_{2}$ variam de acordo com o tipo de pirólise [11-14].

Em relação à composição química dos GC, compõem uma mistura de $30 \%$ de água, $30 \%$ de compostos fenólicos, $20 \%$ de aldeídos e cetonas, $15 \%$ de álcoois e 5\% de compostos diversos [15].

No âmbito de Pesquisa e Desenvolvimento da ANEEL, a CEMIG, em conjunto com empresas produtoras de carvão vegetal como a ONDATEC, investe em projetos de utilização de gases de carbonização que além de contribuirem em eficiência energética, possuem um impacto de mitigação considerável na emissão de gases do efeito estufa. Há também a possibilidade do uso dos gases não condensáveis com potencial de geração, que aumenta 0 aproveitamento dos resíduos do processo. Todo esse esforço considera-se a obtenção de créditos de carbono, aumento da viabilidade técnica da carbonização da madeira pelo processo e aumento da micro e mini geração no estado.

\section{MATERIAIS E MÉTODOS}

Para o estudo do potencial energético dos efluentes da pirólise (carbonização) aqui proposto, foi utilizado como matéria-prima o eucalipto citriodora. O sistema (forno) de carbonização utilizado foi construído pela ONDATEC - Tecnologia Industrial em Micro-ondas, empresa situada em Uberaba no Triângulo Mineiro. O forno utilizado é constituído por uma esteira de alimentação de cavacos, uma balança de alimentação, uma cavidade de secagem por micro-ondas, uma cavidade de pirólise por micro-ondas e um silo de armazenamento de carvão, conforme pode ser observado na Figura 1.

A biomassa foi enfornada em um processo contínuo e após estabelecimento do regime no processo, iniciou-se a amostragem dos efluentes do processo. 


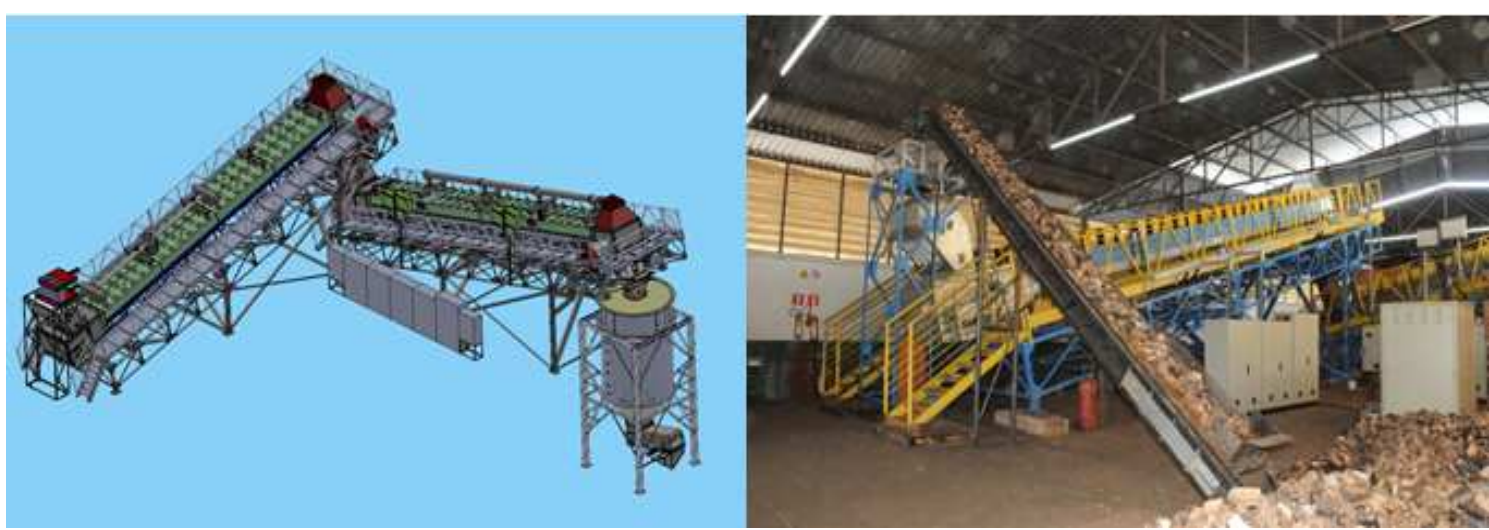

Figura 1. Sistema de carbonização por micro-ondas.

\subsection{Instalação da Estrutura Laboratorial}

Com o objetivo de quantificar as emissões, os gases de exaustão do processo de carbonização foram periodicamente coletados, sendo que na sua fração não condensável, foram analisados no local, por cromatografia gasosa e na sua fração condensável foram coletadas e armazenadas para posterior análise da sua composição química, por meio de cromatografia gasosa acoplada a espectrômetro de massa. A amostragem da fração não condensável foi realizada na chaminé do forno, coletando-se periodicamente os gases da carbonização.

O trabalho envolveu a determinação direta do poder calorífico, por calorimetria, de amostras da madeira enfornada, carvão vegetal produzido e alcatrão coletado. Para determinação do poder calorífico total da fração não condensável dos gases emanados durante o período de carbonização, foram ponderados os poderes caloríficos individuais de seus componentes principais $\left(\mathrm{H}_{2}, \mathrm{CH}_{4}, \mathrm{CO}\right)$. O poder calorífico da fração condensável dos gases (licor pirolenhoso) foi calculado, tomando-se como base o balanço de massa e a energia envolvida no processo de carbonização. A Tabela 1 resume a sistemática de avaliação aplicada no estudo.

Tabela 1. Sistemática de avaliação aplicada ao estudo

\begin{tabular}{|c|c|c|c|c|c|}
\hline & \multicolumn{5}{|c|}{ Componente } \\
\hline & Madeira & Carvão & GNC & GC & Alcatrão \\
\hline Calorimetria & 3 amostras & 3 amostras & ** & *** & AC4 \\
\hline $\begin{array}{l}\text { Espectrometria de } \\
\text { massa }\end{array}$ & --- & --- & --- & AC4 & --- \\
\hline Cromatografia gasosa & --- & --- & $\mathrm{AC} 4$ & --- & --- \\
\hline Pesagem & 3 amostras & 3 amostras & $\#$ & AC4 & AC4 \\
\hline
\end{tabular}

As atividades de coleta de dados foram realizadas nas instalações da ONDATEC, e os resultados deste trabalho deverão subsidiar tecnicamente 0 setor de produção de carvão vegetal e ferro-gusa quanto ao conhecimento dos balanços de massa e do potencial energético dos gases liberados durante a 
carbonização por micro-ondas. Adicionalmente, serão oferecidos resultados da composição química dos principais integrantes dos gases condensáveis e não condensáveis gerados no processo.

Para o desenvolvimento das análises, os equipamentos foram instalados online com o processo. Instalou-se o aparato de amostragem junto à chaminé do forno, realizando-se a coleta e análise dos gases em períodos de 4 horas. Para evitar erro nas amostragens, as medições se iniciaram após o sistema entrar em seu regime permanente (ou estável) de operação. Manteve-se, desse modo, o procedimento padrão de operação do sistema de carbonização durante a coleta dos gases.

Toda a matéria-prima a ser enfornada (toletes de madeira) foi pesada, bem como se procedeu à tomada de amostras para a determinação de seu teor de umidade. O aparato de amostragem de gases (Figura 2), instalado junto à chaminé do forno, foi calibrado para coleta precisa de gases, sendo programado para realizar 5 sessões de amostragem com duração de 10 minutos por hora, a uma taxa de 1,5 L/minuto. $O$ gás coletado a cada ciclo de 4 horas (acumulando-se 5 sessões de amostragem com duração de 10 minutos) foi armazenado em um gasômetro com capacidade de $60 \mathrm{~L}$.

A cada intervalo de 4 horas, uma amostra da fração dos GNC contida no gasômetro foi coletada em bolsas de Tedlar. Neste período, procedeu-se também a limpeza e substituição da vidraria e dos filtros do aparato de amostragem. A análise química do GNC amostrado foi então conduzida por meio de um cromatógrafo gasoso equipado com detector de condutividade térmica. Assim, mediante cromatografia gasosa, determinou-se, no local, a composição e quantidade de cada componente dos GNC.

$\mathrm{Na}$ campanha de amostragem, foi ainda conduzido um procedimento para a coleta da fração de vapores pirolenhosos condensáveis, que foram obtidos pela ação dos condensadores e armazenados nos Erlenmeyers do aparato de amostragem (Figura 2, C1 e C2), para pesagem e posteriores análises químicas em laboratório externo.

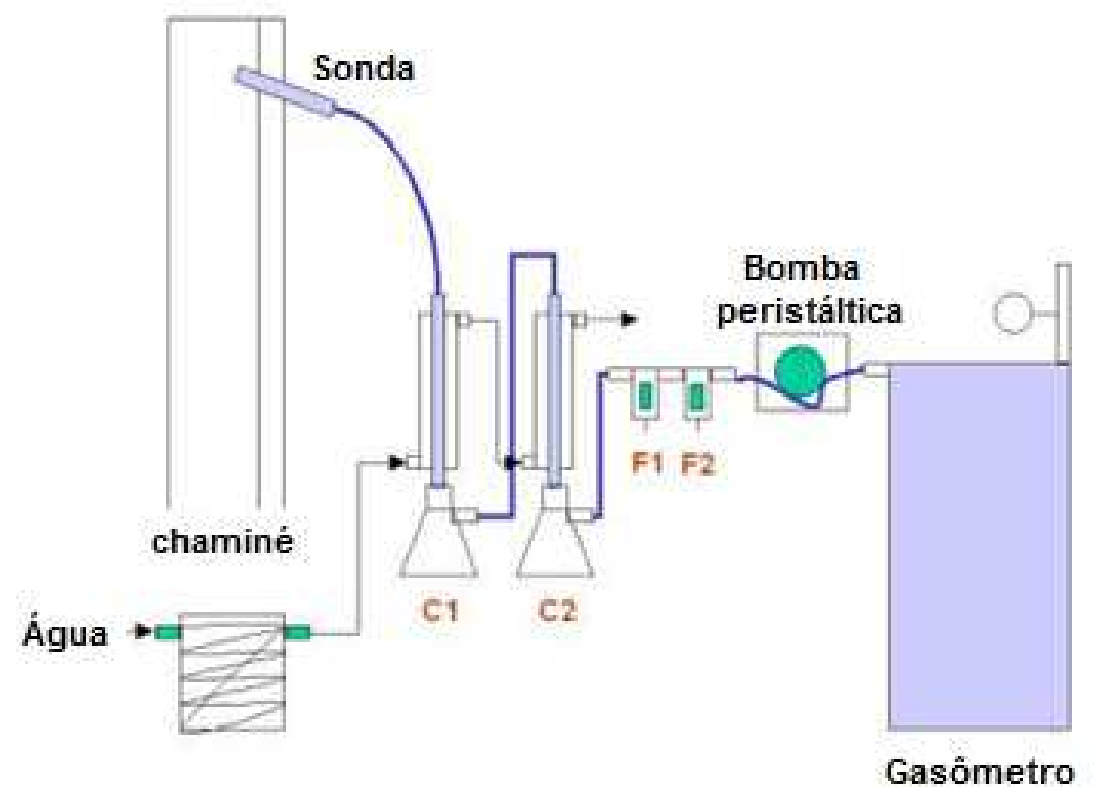

Figura 2. Layout do aparato de amostragem de gases utilizado no experimento conduzido nas instalações da ONDATEC em Uberaba (MG). 
Após a finalização do período de amostragem, o rendimento gravimétrico da carbonização pôde ser calculado a partir da massa seca da carga de madeira e carvão vegetal produzido. Todos os procedimentos de amostragem e cálculos do balanço de massa foram conduzidos com base em adaptação à norma UNFCCC AM0041versão 1 [16].

\subsection{Análises Laboratoriais}

Além da coleta de gases em campo e cromatografia gasosa do GNC do vapor pirolenhoso, o trabalho também envolveu a determinação direta do poder calorífico, por meio de bomba calorimétrica, de amostras da madeira enfornada, carvão vegetal produzido e alcatrão coletado. Para determinação do poder calorífico total do GNC dos gases emanados durante a carbonização, foram ponderados os poderes caloríficos individuais de seus componentes principais $\left(\mathrm{H}_{2}, \mathrm{CH}_{4}, \mathrm{CO}\right)$. O poder calorífico da fração condensável dos gases de exaustão (licor pirolenhoso) foi estimado tomando-se como base o balanço de massa e energia do processo de carbonização.

$\mathrm{Na}$ sequência, foi analisada a composição química do licor pirolenhoso, por meio de cromatografia gasosa acoplada a espectrometria de massa - GCMS.

\section{RESULTADOS E DISCUSSÃO}

Os resultados referentes aos balanços de massa e energia do processo de pirólise de biomassa por micro-ondas na produção de carvão são apresentados em seus valores médios de cinco ensaios realizados nas Tabelas 2, 3 e 4.

Tabela 2. Massas médias de madeira enfornada e carvão produzido

\begin{tabular}{cc|cc}
\hline \multicolumn{2}{c|}{ Matéria-prima enfornada } & \multicolumn{2}{c}{ Produtos sólidos gerados } \\
\hline $\mathrm{M}_{\text {madeira alimentada }}$ & $3043 \mathrm{~kg}$ & $\mathrm{M}_{\text {carvão úmido }}$ & $1072 \mathrm{~kg}$ \\
\hline $\mathrm{M}_{\text {madeira seca }}$ & $2878 \mathrm{~kg}$ & $\mathrm{M}_{\text {carvão seco }}$ & $1061 \mathrm{~kg}$ \\
\hline Umidade $\mathrm{BC}$ & $5,70 \%$ & Umidade $\mathrm{BC}$ & $1,00 \%$ \\
\hline & & $\mathrm{M}_{\text {tiço }}$ & $62 \mathrm{~kg}$ \\
\hline
\end{tabular}

Tabela 3. Balanços de massa médios dos GNC e suas frações

\begin{tabular}{ccc}
\hline \multicolumn{2}{c}{ Massas de GNC [kg] } & Proporções (\%) \\
\hline Massa de $\mathrm{H}_{2}$ & 1 & 0,04 \\
\hline Massa de $\mathrm{O}_{2}$ & 225 & 9,54 \\
\hline Massa de $\mathrm{N}_{2}$ & 1085 & 46,01 \\
\hline Massa de $\mathrm{CO}_{2}$ & 704 & 29,86 \\
\hline Massa de $\mathrm{CO}$ & 283 & 12 \\
\hline Massa de $\mathrm{CH}_{4}$ & 60 & 2,54 \\
\hline Massa total de $\mathrm{GNC}$ & 2358 & \\
\hline
\end{tabular}


Tabela 4. Balanços de massas médios dos GC e suas frações

\begin{tabular}{|c|c|c|c|}
\hline Item & Composto & Massa total média kg & Proporção \% \\
\hline 1 & Metanol & 30,7 & 3 \\
\hline 2 & Acetona & 3,8 & 0,37 \\
\hline 3 & Acetato de metila & 18,1 & 1,77 \\
\hline 4 & 2,3-Butanenodiona & 7,5 & 0,73 \\
\hline 5 & 2-Butanona & 2,1 & 0,21 \\
\hline 6 & Ácido acético & 106,7 & 10,42 \\
\hline 7 & Acetol & 17,6 & 1,72 \\
\hline 8 & Butanenodiol & 3,3 & 0,32 \\
\hline 9 & Furaldeído & 37,2 & 3,63 \\
\hline 10 & 2-Furanmetanol & 8,5 & 0,83 \\
\hline 11 & 2-Propanona, 1-hidróxi-acetato & 3,5 & 0,34 \\
\hline 12 & Dimetoxitetrahidrofurano & 4,8 & 0,47 \\
\hline 13 & 2-Metil, 2-ciclopentenona & 4,4 & 0,43 \\
\hline 14 & Etanona, 1-(2-furanil)- & 1,8 & 0,18 \\
\hline 15 & 1-2-Ciclopentanediona & 5,9 & 0,58 \\
\hline 16 & 2-Furancarboxialdeído, 5-metil & 14,4 & 1,41 \\
\hline 17 & Corilon & 13,9 & 1,36 \\
\hline 18 & p-Cresol & 9,8 & 0,96 \\
\hline 19 & 2-Metoxi fenol & 20,5 & 2 \\
\hline 20 & 4-Metoxi-3-metilfenol & 1,7 & 0,17 \\
\hline 21 & P-Cresol. 2-metoxi & 24,7 & 2,41 \\
\hline 22 & 3-Metoxi-1,2-benzenediol & 21,7 & 2,12 \\
\hline 23 & 4-Etil 2-metoxi fenol & 6,9 & 0,67 \\
\hline 24 & 4-Metil-1,2-benzenediol & 2,5 & 0,24 \\
\hline 25 & 2,6-Dimetoxifenol & 37,3 & 3,64 \\
\hline 26 & 1,2,4-Trimetoxibenzeno & 25 & 2,44 \\
\hline 27 & 1,2,3-Trimetoxi-5-metil-benzeno & 29,5 & 2,88 \\
\hline 28 & Alcatrão & 139,5 & 13,62 \\
\hline 29 & Água & 337,5 & 32,96 \\
\hline \multirow[t]{2}{*}{30} & Outros & 83,2 & 8,13 \\
\hline & Massa total & 1024 & - \\
\hline
\end{tabular}

$\mathrm{Na}$ Tabela 5 e na Tabela 6 são apresentados os resultados da composição dos GC e GNC e seus respectivos valores de poder calorífico inferior (PCl).

Tabela 5. Composição Gás condensável (GC) (pirolenhoso + Alcatrão)

\begin{tabular}{cccc}
\hline \multicolumn{4}{c}{ Composição Gás condensável (pirolenhoso + Alcatrão) } \\
\hline \multirow{2}{*}{ Gases } & $\begin{array}{c}\text { Massa Medida } \\
\mathbf{k g}\end{array}$ & $\% \mathbf{p} / \mathbf{p}$ & Energia (MJ) \\
\hline Pirolenhoso & 463,8 & 45,29 & 13016 \\
\hline $\mathrm{N} / \mathrm{D}$ & 83,2 & 8,13 & $\mathrm{~N} / \mathrm{A}$ \\
\hline Água & 337,5 & 32,96 & $\mathrm{~N} / \mathrm{A}$ \\
\hline Alcatrão & 139,5 & 13,62 & 3125 \\
\hline Massa de gás & 1024 & & 16141 \\
\hline & & $\mathbf{P C l}(\mathbf{M J} / \mathbf{k g})$ & $\mathbf{1 5 , 7 6}$ \\
\cline { 3 - 4 } & &
\end{tabular}


Tabela 6. Composição Gás não condensável (GNC)

\begin{tabular}{cccc}
\hline \multicolumn{5}{c}{ Composição Gás não condensável (GNC) } \\
\hline Gases & Massa kg & $\%$ p/p & Energia PCl \\
\hline $\mathrm{H}_{2}$ & 1 & 0,04 & 120 \\
\hline $\mathrm{O}_{2}$ & 225 & 9,54 & $\mathrm{~N} / \mathrm{A}$ \\
\hline $\mathrm{N}_{2}$ & 1085 & 46,01 & $\mathrm{~N} / \mathrm{A}$ \\
\hline $\mathrm{CO}_{2}$ & 704 & 29,86 & $\mathrm{~N} / \mathrm{A}$ \\
\hline $\mathrm{CO}$ & 283 & 12,00 & 2585 \\
\hline $\mathrm{CH}_{4}$ & 60 & 2,54 & 3000 \\
\hline Massa de gás & 2358 & & 5705 \\
\hline & & $\mathbf{P C l}(\mathbf{M J} / \mathbf{k g})$ & $\mathbf{2 , 4 2}$
\end{tabular}

Pode-se observar que o poder calorífico inferior dos GC pode ser aumentado com a retirada da água de sua composição e que o baixo valor do $\mathrm{PCI}$ no $\mathrm{GNC}$ se justifica pela presença significativa de $\mathrm{O}_{2}, \mathrm{~N}_{2}, \mathrm{CO}_{2}$, presentes nesse gás. Esses compostos se devem principalmente à entrada de ar no forno durante 0 processo e a não retirada de vapores de água no protótipo.

Ao efetuar o balanço de massa no protótipo (valores médios), observou-se uma diferença de $1638 \mathrm{~kg}$ entre as massas de entrada e saída do forno de carbonização, essa diferença se deve a entrada de ar no interior no forno (protótipo). A entrada de ar no interior do forno implica em vários inconvenientes, principalmente em riscos de explosão, porém no que se relaciona às reações químicas na pirólise da biomassa, tem-se a oxidação do carbono (combustão), formando, dentre outros, $\mathrm{CO}_{2}$.

O estudo da disponibilidade energética aqui efetuado, apresentou uma disponibilidade média de 5,60 MWh de energia térmica por tonelada de carvão vegetal produzido pela tecnologia ONDATEC.

Com a utilização de um sistema de geração de energia com rendimento de $40 \%$, pode-se obter $2,24 \mathrm{MWh}$ de energia elétrica por tonelada de carvão. $\mathrm{O}$ consumo para a produção de uma tonelada de carvão no protótipo é de 1,4 MWh de energia elétrica, com a geração de 2,24 MWh, ter-se-á o excedente de $0,84 \mathrm{MWh} /$ ton $_{\text {carvão. }}$

Sabe-se que nos fornos convencionais mais de $75 \%$ da massa seca da madeira enfornada é transformada em gases que são lançados diretamente na atmosfera sem qualquer controle. Desta forma, a mudança de tecnologia para esta apresentada neste paper eliminará a poluição que existe no processo convencional e produzirá energia limpa e renovável.

$\mathrm{O}$ investimento para este sistema gira em torno de $\mathrm{R} \$ 1.000 .000,00$. Ao considerar o valor médio do $\mathrm{MWh}$ a $\mathrm{R} \$ 313,16$; o excedente energético de 0,84

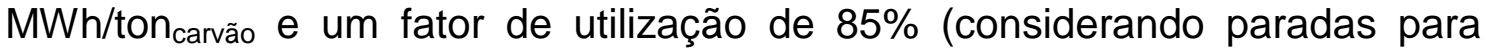
manutenção) o investimento é recuperado em aproximadamente 3 anos e meio.

\section{CONCLUSÃO}

Pode-se concluir ante ao exposto que a tecnologia desenvolvida e aqui mostrada é uma boa alternativa para o melhor aproveitamento da biomassa das florestas destinadas a produção de carvão vegetal. Há no estado de Minas Gerais, bem como no país, um potencial de aproveitamento da energia do processo de produção de carvão vegetal muito grande. 
Demonstrou-se que as contribuições não serão apenas no âmbito da possibilidade de geração excedente de 0,84 MWh por tonelada de carvão produzido, mas para o meio ambiente e para a qualidade de vida das pessoas envolvidas na sua cadeia produtiva.

Essa tecnologia pode contribuir muito para desoneração da cadeia produtiva do ferro-gusa no Brasil.

\section{Agradecimentos}

Agradecemos à CEMIG - Companhia Energética de Minas Gerais, pelas bolsas cedidas e pelo apoio financeiro e ao Programa de Pesquisa e Desenvolvimento ANEEL e a REDEMAT.

\section{REFERÊNCIAS}

1 Brito, J.O. O uso energético da madeira.Estudos avançados 21(59).9 páginas.

Escola Superior de Agricultura (Esalq/USP).(2007)

2 Meenakshi, P. Elements of Environmental Science and Engineering - pg 227; 237 - Ed. Prentice Hall, Delhi 312p., 2008

3 Thiago, P.P; Alves,I.C.N; Trugilho, P.F; Silva, V.O; Baliza, A.E.R.Compactação de biomassa vegetal visando à produção de biocombustiveis sólidos. Brazilian Journal of Foresty Research.12 páginas (2010).

4 Braga, N.P. Quantidade e qualidade das emissões atmosféricas na carbonização da madeira. II Forum nacional sobre carvão vegetal.24 páginas, 2010. Arcelor Mittal Bioenergia.

5 Companhia Energética de Minas Gerais; $28^{\circ}$ Balanço Energético do Estado de Minas Gerais; Belo Horizonte, 2014.

6 Pereira, E.J.S; Pinho, J.T. Forno industrial de micro-ondas de $3 \mathrm{~kW}$ alimentado por magnetrons de uso domestico. Grupo de Estudos e Desenvolvimento de Alternativas Energéticas - GEDAE/DEEC/CT/UFPA. 5 páginas. 2000

7 Fernández, Y; Arenillas, A; Menéndez, Á. Microwave Heating Applied to Pyrolysis. Instituto Nacional del Cárbon, 30 páginas, 2011. DOI: 10.5772/13548

8 Trugilho, et al; Energia de Biomassa Florestal: A Contribuição da UFLA. Publicado em Renabio: Biomassa\&Energia, v. 1, n. 3, p. 221-224, 2004.

9 Browning, B.L. The chemistry of wood. Publicado em New York, John Wiley \& Sons. p. 689, 1963.

10 PetterseN, R.C. The chemical composition of wood. In: ROWELL, R. (ed.) The chemistry of solid wood. Published in Washington, American Chemical Society. p.54-126, 1984.

11 Scott, D. S.;Piskorz, J. The flash pyrolysis of aspen-poplar wood. Publicado em The Canadian Journal of Chemical Engineering, $n^{\circ}$ 60. p. 666-74, 1982.

12 Scott, D. S.; PiskorZ, J. The continuous flash pyrolysis of biomass. Publicado em The Canadian Journal of Chemical Engineering, n62. p.404-12, 1984.

13 Scott, D. S.; Piskorz, J.; Bergougnou, M. A; Graham, R.; Overened, R. P. The role of temperature in the fast pyrolysis of cellulose and wood. Publicado em The Canadian Journal of Chemical Engineering, $n^{\circ} 27$. p.8-15, 1988.

14 Horne, P. A.; Williams, P.T. Influence of temperature on the products from the flash pyrolysis of biomass. Publicado em Fuel n75. p.1051-9,1996.

15 Fernando, S.; Adhikari, S.; Chandrapal, C.; Murali, N. Biorefineries: current status, challenges, and future direction. Publicado em Energy Fuels, 2006;20:1727-37.

16 United Nations Framework Convention on Climate Change. Approved baseline and monitoring methodology AM0041 "Mitigation of Methane emissions in the wood carbonization activity for charcoal production": version 01. 2006. 63p. Disponível em: http://www.mct.gov.br/upd blog/0014/14282.pdf. Acesso em: 07 agosto de 2013. 